Research Article

\title{
The Image Analysis of the Visual Sensor and the Effect of Vitamin E Supplementation on Football Players' Free Radicals and Their Physical Strength Supplement
}

\author{
Wei Zhao \\ College of Physical Education, Shaanxi University of Technology, Hanzhong, 723000 Shaanxi, China \\ Correspondence should be addressed to Wei Zhao; zhaowei@snut.edu.cn
}

Received 26 August 2021; Revised 1 November 2021; Accepted 5 November 2021; Published 28 November 2021

Academic Editor: Haibin Lv

Copyright (C) 2021 Wei Zhao. This is an open access article distributed under the Creative Commons Attribution License, which permits unrestricted use, distribution, and reproduction in any medium, provided the original work is properly cited.

\begin{abstract}
With the help of image analysis technology of visual sensors, the research discusses the supplementation of vitamin E on free radicals and physical strength of football players after intensive exercise. The free radical supplementation and physical strength supplementation of athletes after strenuous exercise by taking a proper amount of vitamin E were studied and discussed. The effects of vitamin $\mathrm{E}$ on free radical metabolism, serum antioxidant level, and antioxidant enzyme activity and serum lipid peroxide metabolism after acute exercise can be generalized. 40 football players were recruited from the sports department of a physical education college in our province and randomly divided into the experimental group and the control group. The experimental group was divided into the $\mathrm{T} 1$ group and the $\mathrm{T} 2$ group, and the control group was also divided into the $\mathrm{C} 1$ group and the $\mathrm{C} 2$ group accordingly, with 10 players in each group. After the football league, the $\mathrm{C} 1$ and $\mathrm{T} 1$ groups will perform maximal aerobic exercise, and the $\mathrm{C} 2$ and $\mathrm{T} 2$ groups will perform intermittent anaerobic exercise. Serum malondialdehyde (MDA), total antioxidant capacity (T-AOC), glutathione peroxidase (GSH-PX), and superoxide dismutase (SOD) were determined. The comparison between the control group taking placebo and the experimental group taking vitamin E for a period of time shows that the average values of MDA are $3.25 \pm 0.31(\mathrm{~nm} / \mathrm{ml})$ and $4.20 \pm 0.78(\mathrm{~nm} / \mathrm{ml})$, respectively. After taking vitamin E, the antioxidant level of the experimental group was significantly higher than that of the control group, serum antioxidant enzyme activity decreased, serum lipid peroxidation products decreased significantly, and more free radicals were generated immediately after aerobic exercise. Vitamin E can significantly reduce the generation of free radicals during football players' intermittent anaerobic exercise and improve the body's antioxidant capacity.
\end{abstract}

\section{Introduction}

In recent years, more and more sports professionals have started to achieve better results in more intense professional training, such as extreme sports, triathlon, and Hercules competitions. The formation of this kind of ethos has also formed a demonstration role for the folk fitness exercise. It is also more and more common for the folk to carry out some irregular intense acute exercises. If these exercises cannot be carried out scientifically, they will cause different degrees of damage to the public body. The cause of the injury is that in acute high-intensity exercise, the body will produce oxygen free radicals and cause physical damage to the body. At present, this issue is being paid attention to by people in the field of sports medicine, and a lot of research experiments have been carried out.

Since the last century, biologists have explored the relationship between free radicals and clinical diseases. With the development of social technology and the improvement of free radical research technology, it has been found that the production mechanism of exercisable endogenous free radicals also includes neutrophil mechanism, prostaglandin mechanism, and calcium mechanism [1]. The mitochondrial mechanism and xanthine oxidase mechanism are generally considered two mechanisms for the production of sportive endogenous free radicals [2]. Modern medical planners are more inclined to believe that free radicals are the cause of many diseases in the body and that acute intense exercise 
produces a large number of free radicals [3]. Exercise induced endogenous free radicals to rats as experimental object in establishing an exercise fatigue model, with increased rat liver and skeletal muscle after exercise and myocardial mitochondria change on superoxide anion; rats increased load exhaustion after exercise, it is found that the rat's liver increased significantly on superoxide anion, and skeletal muscle mitochondria are significantly higher on superoxide anion, but no other significant changes occurred $[4,5]$. In the past research, it has been confirmed that the generation of free radicals can increase with the increase of oxygen consumption; if free radicals in the body cannot be effectively cleared in time, it can cause tissue cell membrane unsaturated fatty acid attack, increase the permeability of cell membrane, and cause protein leak and cell necrosis [6].

At present, based on the available data and the pathway of endogenous free radical generation in exercise, the level of free radical metabolism and the degree of lipid peroxidation in exercise are reflected by the generation of tissue free radicals and lipid peroxidation after acute exercise [7]. There was no mitochondrial respiration being the pathway of increased myocardial free radical production during exercise, and the perception of potassium cyanide by mitochondria was significantly impaired, presumably being a stimulation of the xanthine oxidase pathway in which hydrogen peroxide production had been significantly increased [8]. It was shown that superoxide anion free radicals formed by electron leakage are the main source of exercisable endogenous free radicals during the respiratory chain and electron transport in mitochondria of liver and skeletal muscle [9]. There are few researches on football and free radicals. This study is through the analysis of college students' football training on football athletes before and after malondialdehyde (MDA), superoxide dismutase (SOD), and glutathione peroxidase (GSH-PX), in order to investigate vitamin $\mathrm{E}$ for football player free radical metabolism and antioxidant enzyme activity level of the effect of vitamin $\mathrm{E}$ and provide certain reference for football teaching and scientific training.

\section{Research Overview}

2.1. Generation of Free Radicals and Metabolism In Vivo. Free radicals are atoms or atomic groups containing one or more unpaired electrons, which are inevitable intermediate products of biochemical reactions in biological cell metabolism that can exist independently in nature [10]. In the body, with more than $95 \%$ of them belonging to oxygen radicals, common oxygen radicals for the body include reactive oxygen species, singlet oxygen species, hydrogen peroxide, oxygen organic radicals, oxoperoxides, and superoxide anions [11]. The generation of free radicals occurs once the biochemical reactions of organism cells during metabolism participate in enzymes and catalyze [12].

There are two basic types of oxygen free radicals: exogenous free radicals and endogenous free radicals. The production of exogenous free radicals is more common in smoking, drinking, unhealthy life, or work habits. When excessive exogenous oxygen free radicals are produced in the body, the antioxidant defense system in the body will be helpless in the face of numerous free radicals, causing cell lipid peroxidation, carbohydrate oxidation, protein denaturation, and enzyme inactivation in the body and even leading to cell canceration. The increase of endogenous free radicals mainly includes catecholamine, phospholipid-arachidonic acid pathway, xanthine oxidase increase, outbreak of "breathing" of phagocytic cells, quinone compounds in the body breakdown, microsomal mixed function oxidase catalysis, somatic cell within some enzymatic reaction, and oxygenated hemoglobin in red blood cells spontaneous transformation into methemoglobin.

\subsection{The Generation Mechanism of Free Radicals Caused by} Exercise. High intensity exercise will cause the production of free radicals. The production of free radicals in exercise mainly includes the following ways.

2.2.1. Neutrophil Mechanism. With intense exercise, not only muscle and skeletal disorganization is involved, but it can also generate similar inflammatory responses in organs including the heart and liver and lead to the aggregation of neutrophils, and when aggregated in large numbers, the catalytic action of oxidase in the hair should directly promote the generation in free radicals.

2.2.2. Xanthine Oxidase. During strenuous exercise, the body is short of energy and ATP is always in short supply. This activates the reaction catalyzed by adenosine kinase, and xanthine oxidase can form aggregation in the body. During the process of xanthine oxidase catalysis, free radicals are produced. High intensity exercise will lead to the accumulation of uric acid in athletes' plasma, and the overload exercise load in a short time will cause the exponential growth of free radicals. Under the action mechanism of ischemiareperfusion, the reaction catalyzed by antioxidant enzymes is the main source of free radicals. When the exercise is over, the blood will automatically restore the normal blood supply to the liver organs. Therefore, during exhaustive exercise training, the activity of antioxidant enzymes will be increased in the muscles, thus increasing the production of free radicals.

2.2.3. Mitochondrial Respiratory Transport Chain. During the ideal state of proper human body, in the respiratory chain, in the electron leakage process, about $1-5 \%$ of oxygen is being transformed into reactive oxygen free radicals by mitochondria, with the vast majority of oxygen free radicals that are generated by coenzyme Q. Among the important enzymes that mitochondria participate in the formation of energy ATP, lactate dehydrogenase and coenzyme Q produce oxygen free radicals. In the overloaded exercise intensity, the oxygen consumption of mitochondria in muscle fibers is as high as 100 times, and the oxygen consumption of the whole body can be increased by 20 times. Therefore, free radicals may be produced in large quantities with the high oxygen consumption of mitochondria in the intense exercise state. 
2.2.4. Calcium Mechanism. The intensity of exercise training will lead to the influx of calcium ions; the increase of calcium ions in cells will cause the deposition of calcium phosphate in mitochondria, aggravate the damage of mitochondrial membrane, and affect the function of mitochondria. Calcium imbalance can affect the release of calcium from the sarcoplasmic reticulum and cause damage to muscle cell membrane, resulting in free radical production. However, this mechanism is not clear at present and needs further study.

\subsection{Movement Mode and Generation of Free Radicals. Exer-} cise in order to achieve a strong body at the same time will cause the body to produce a large number of free radicals. People in the sports medicine research report pointed out that the role of free radicals and the nature of sports, exercise intensity, and the body itself is closely related to the endurance quality; acute chronic exercise may lead to free radicals increases; increase under the condition of lacking the ability to remove free radicals can make the body cells in the oxidative stress state and bring continuous pathological damage to the human body. Many sports medical research report pointed out that the movement of acute and chronic can cause the increase of human free radicals and bring a series of pathological damage to the body, but the free radicals from acute and chronic movement are equivalent, and how to eliminate free radicals caused by movement and promote the recovery of fatigue after exercise has been a difficult point in the field of sports science research. More and more case studies prove that acute exercise and chronic exercise produce more free radicals; acute movement promotes the production of large numbers of free radicals which has two mechanisms: One is that local tissue accumulation of metabolites and hypoxia can cause large amounts of oxygen consumption, which affects the oxidation of mitochondrial function and provides more opportunities for single electron reduction, thus arousing a series of free radicals. Secondly, excessive exercise inevitably brings about a sharp increase in instantaneous oxygen consumed, and oxygen metabolism inevitably leads to the production of oxygen free radicals. Intense sports are characterized by intense competition, fierce rivalry, and intense movement. Because of highintensity sports with many repetitions, the athlete's body becomes more oxygen-depleted and is in a hypoxic state, and the generation of free radicals increases significantly, and this causes cellular damage. Superanion free radicals can be removed by superoxide dismutase (SOD) and glutathione peroxidase (GSH-PX) to protect cells, and the activity of superoxide dismutase (SOD) and glutathione peroxidase (GSH-PX) can indirectly reflect the ability of the body to remove oxygen free radicals.

2.4. Mechanism of Vitamin $E$ in the Body. Vitamin $\mathrm{E}$ is a good natural antioxidant. It is an important part of the biofilm. It mainly exists in the inner mitochondrial membrane of the cell membrane. There are four types of vitamin $\mathrm{E}$ in nature. The group on the sixth carbon atom in its ring structure can provide electrons and is reductive, and the benzene ring in the formula has the ability of electron resonance, which makes the oxidized product of vitamin $\mathrm{E}$ quite stable. Therefore, vitamin $\mathrm{E}$ is an important fat-soluble antioxidant in organisms. It can prevent the lipid peroxidation chain reaction caused by oxygen free radicals and block the action of free radicals on the membrane of polyvalent unsaturated fatty acids to protect the integrity of cells, and vitamin E can react with singlet oxygen to prevent the damage of singlet oxygen to cells.

Vitamin $\mathrm{E}$ is a fat-soluble vitamin and an important nutrient for the human body. Vitamin $\mathrm{E}$ is an excellent scavenger with a strong antioxidant effect. It plays an important role in prevention and treatment of DNA damage, chromosomal malformation, DNA admixture and micronucleus formation, cell damage and death, and maintenance of cell DNA stability caused by reactive oxygen species. Therefore, how sports can cooperate with sports nutrition to achieve fitness and improve performance is worthy of attention and in-depth study. The purpose of this study is to understand the oxidative protection effect of lycopene supplementation on the body after acute intense exercise, so as to provide some useful references for sports training, sports fitness, health care, and injury prevention.

2.5. Image Analysis of Vision Sensors. By recording the actions of football players in the course of exercise and analyzing the connection between actions and free radicals, it is possible to propose reforms to the actions of football players based on the experimental results and to obtain optimal results from various aspects such as technical improvement and sports safety. The image processing of the vision sensor is applied in football sports as shown in Figure 1.

In the image recording process, if the human body is divided into left and right sides to perform the spatial dynamic calculation of the visual sensor, there will be

$$
\begin{aligned}
& A(l, r)=\frac{\mid \text { posture }(l, r) \mid}{\sqrt{l \otimes \sqrt{r}}} *(l, r), \\
& \left(l, l^{\prime}\right)=\frac{1}{i} \sum_{i=1}^{m}\left\|f_{i}(l)-f_{i}\left(l^{\prime}\right)\right\| .
\end{aligned}
$$

Among them, $A(l, r)$ is the recorded overall spatial posture of the athlete's body, $l$ is the left side, and $r$ is the right side; formula (2) is the estimation error value of a single part of the human body posture, the recorded error is the $m$ th error point in the human body, and $f_{i}$ is a certain point of the extraction error. Tracking the error can be expressed as

$$
\begin{aligned}
\left(a, a^{\prime}\right) & =\frac{1}{s} \sum_{i=1}^{s}\left|\left(a_{i}-a_{i}^{\prime}\right) \bmod \mp 180^{\circ}\right|, \\
\beta_{a} & =\sqrt{\sum_{i=1}^{n}\left\|A_{n, a}^{(i)}\right\|^{2}}+\alpha^{2} .
\end{aligned}
$$




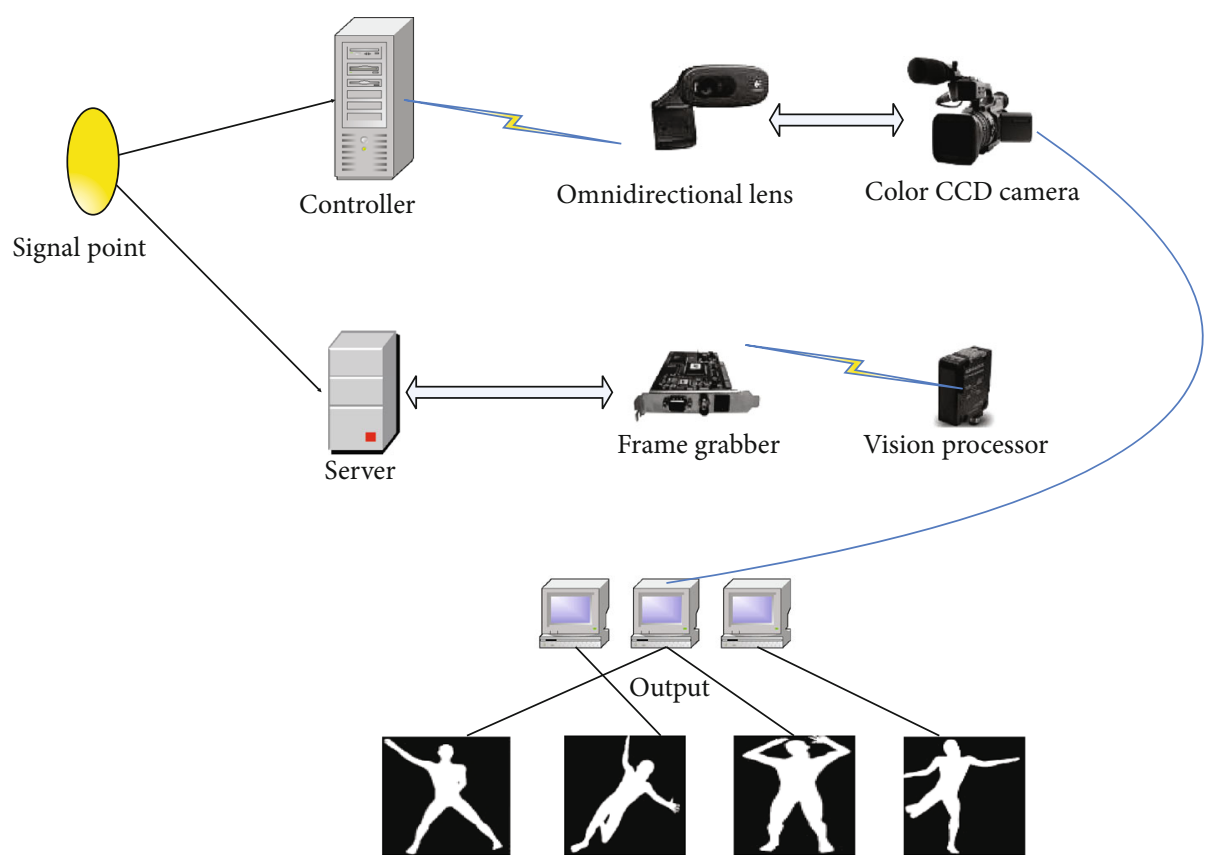

FIgURE 1: Vision sensor applied to the recording of football sports.

Through constraint $0<\alpha<1$, the following questions about tracking optimization can be obtained:

$$
\begin{aligned}
a_{i}^{n} & =f\left(\beta_{i}^{n-1} ; N^{*}, b^{*}\right)<B_{i}^{n}+1, \\
F_{i}\left(f, r, r_{1}, \varepsilon\right) & = \begin{cases}1, & \text { other, } \\
o, & \text { if } \vartheta^{f}(r)<\vartheta^{f}\left(r_{1}\right)+\varepsilon .\end{cases}
\end{aligned}
$$

$F_{i}$ is obtained by comparing the two tracking feature values in the dichotomous test, which can be applied to the state prediction of an internal model of a specific behavior. In order to realize the time transition between different postures, according to the multiview image sequence, it is calculated as

$$
\begin{gathered}
\sin l_{i}^{c}=\sin <\chi_{t}^{i}, \chi_{t-1}^{i} \geq \chi_{t}^{i} \cdot \chi_{t-1}^{i}, \\
\chi_{t}^{*}=\frac{\left(\chi_{t}^{i-1}-\chi_{t-1}^{i}\right)-\chi_{t-2}^{i}}{\left\|\chi_{t}^{i}\right\| \otimes\left\|\chi_{t-1}^{i}\right\|} .
\end{gathered}
$$

The above formula is calculated on the basis of $t \pm \varepsilon$, and $\chi_{t}^{i}$ is the angle of rotation of a certain point on the posture during movement. $l_{i}^{c}$ is the second derivative. During the whole movement process, the largest change in amplitude is the end point of the body. If a transition bridge is established to the end point, there is

$$
\begin{aligned}
\left(f^{*}, g^{*}\right) & =\min _{(f, g)} \sum_{i \in(l, r)}\left\|\mu_{i}(f, g)\right\|, \\
E(\mu) & =\sum_{A=0}^{I-1} Q_{\mu}(A) \log Q_{\mu}(A),
\end{aligned}
$$

$$
Q_{\mu}(A)=\frac{1}{\|\mu\|} \sum_{a \in \mu}\left(A_{i}=A\right) .
$$

In order to maximize the image information of the endpoint, namely, $\left(f^{*}, g^{*}\right)$, the entropy $\mu$ is fully divided; $E(\mu)$ is the proportion of the sample, which is $E(\mu)=0$ at the endpoint. The confidence value of all joints in the image under the two-dimensional coordinates is defined as

$$
\begin{aligned}
I(u \mid p) & =\prod_{a} I\left(u_{a} \mid p_{a}\right) \eta_{a}, \\
I & =\left(u_{a} \mid p_{a}\right) \infty \eta_{a}\left(u_{a}, i_{a}\right), \\
\max _{p} I(p \mid u) & =\max _{p}\left[\sum_{a} \ln \left(u_{a}+I(p)\right)\right] .
\end{aligned}
$$

The two-dimensional pixel coordinate of the ath limb joint is $u_{a}, p$ represents the two-dimensional posture of the human body, $I$ represents the independent condition of the image generated by the joint [13], and the most likely state of the appearance of the image and the joint is obtained by equation (9). For this, the approximate value is applied to the joint feature vector:

$$
\frac{\phi^{2} f_{v}}{\phi i^{l} \phi i^{r}} \mid i_{n-1} \cong \sum_{V=1}^{I} \theta_{v k} .
$$

Use the linear feature fitting formula to get

$$
K^{(i)}(l)=f_{k}\left(l_{p}\right)+\sum_{v=1}^{w} i^{l} i^{r},
$$



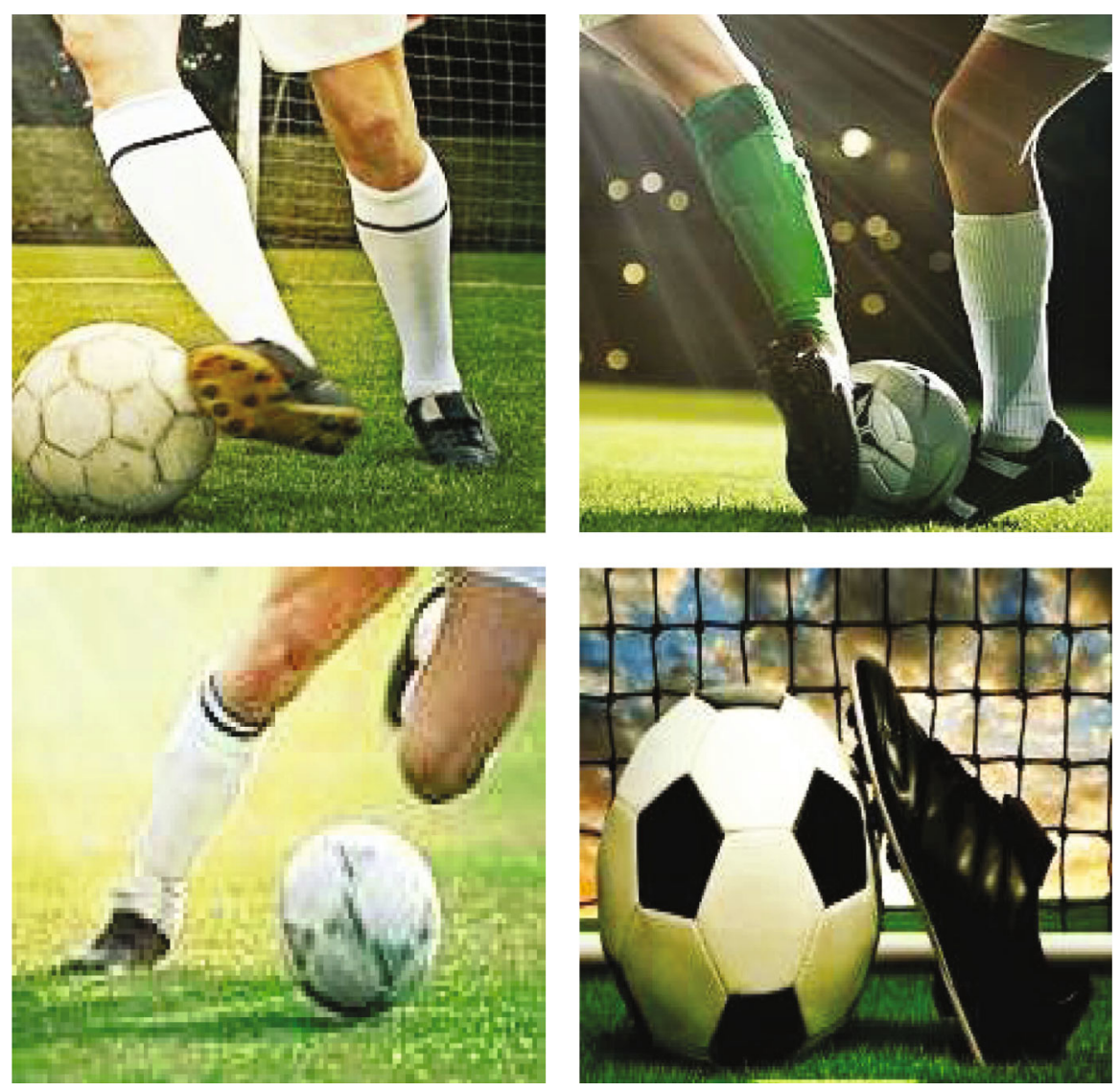

Figure 2: Football.

$$
L_{i}=-\frac{f_{k}}{i^{u}} \mid\left(\frac{\lambda k^{2}}{i(v-u)}-\frac{1}{2}\right) .
$$

Operator $K$ is obtained by a fitting polynomial, and any polynomial $L_{i}$ tends to 0 under the constraint of function $f_{k}$. Using the retrieval pose for weighted solution, you can get

$$
\begin{gathered}
\therefore \delta_{i}=\frac{1}{\sqrt{l}} \frac{\left[\sin \left(\delta_{i}\right), \cos \left(\delta_{i}\right)\right]}{r}, \\
\forall_{i}^{(r)} f\left(s_{i}\right)+\varsigma_{1}\left(t_{i}^{(r)}\right)=0, \\
\forall_{i}^{l} f\left(s_{i}\right)-\because t_{i}(l-1) \leq \varsigma_{1} .
\end{gathered}
$$

$S_{i}$ is a nonzero coefficient. The amplitude matrix spanned by the action is $\forall_{i}^{r}, \forall_{i}^{l}$.

\section{Experimental Design}

3.1. Experimental Subjects. Football (Figure 2) has always been a sport with a high degree of worldwide attention. Since futsal was run by the International Football Association in 1989, it has quickly attracted the attention of the world, prompting more and more countries and football enthusiasts to devote themselves to it. In football, the physical fitness of football players has always been a concern.
Forty football players were selected from the sports department of a sports college in our province and randomly divided into an experimental group and a control group. The experimental group was divided into the $\mathrm{T} 1$ group and the T2 group, and the control group was correspondingly divided into the $\mathrm{C} 1$ group and the $\mathrm{C} 2$ group, with 10 players in each group. The average body weight of the T1 group was $71.4 \pm 10.4 \mathrm{~kg}$, the average height was $175.2 \pm 11.5 \mathrm{~cm}$, and the average age was $20 \pm 3$ years. The average weight of the T2 group was $72.6 \pm 8.3 \mathrm{~kg}$, the average height was $177.2 \pm$ $12.6 \mathrm{~cm}$, and the average age was $20 \pm 4$ years. The average weight of the $\mathrm{C} 1$ group was $70.5 \pm 12.6 \mathrm{~kg}$, the average height was $177.2 \pm 9.5 \mathrm{~cm}$, and the average age was $19 \pm 3$ years. In the C2 group, the average weight was $70.8 \pm 11.5$ $\mathrm{kg}$, the average height was $175.8 \pm 10.2 \mathrm{~cm}$, and the average age was $21 \pm 3$ years. All subjects had to undergo rigorous screening to exclude those with major digestive diseases, upper respiratory infections, or prior medical history, and all had to meet recent requirements for free radical scavengers such as vitamin E. The specific conditions of the research objects are shown in Table 1.

3.2. Experimental Scheme. Intensive training will begin 10 weeks before the start of the provincial football league. The duration of intensive training is initially set at 10 weeks, and further adjustment can be made at any time with the overall situation of the training. The training is divided into 
TABLE 1: The specific situation of the research object.

\begin{tabular}{lccccc}
\hline Group & $n$ & Weight $(\mathrm{kg})$ & Height $(\mathrm{cm})$ & Age (years) & Football practice (years) \\
\hline T1 & 10 & $71.4 \pm 10.4$ & $175.2 \pm 11.5$ & $20 \pm 3$ & $2.4 \pm 0.3$ \\
T2 & 10 & $72.6 \pm 8.3$ & $177.2 \pm 12.6$ & $20 \pm 4$ & $2.8 \pm 0.3$ \\
C1 & 10 & $70.5 \pm 12.6$ & $177.2 \pm 9.5$ & $19 \pm 3$ & $3.1 \pm 0.6$ \\
C2 & 10 & $70.8 \pm 11.5$ & $175.8 \pm 10.2$ & $21 \pm 3$ & $2.7 \pm 0.4$ \\
\hline
\end{tabular}

three stages. The first stage is to improve the basic skills and physical qualities of the subjects. The focus of the second stage is to improve the practice level of the athletes. The third stage is the process of testing the practical effect and filling the gaps. The training content is the process of athletes' physical training and combining physical strength with football skills, including coordination, agility, endurance, and strength training, such as $20 \mathrm{~m}$ frog jump, $30 \mathrm{~m}$ acceleration run, $4 \times 10 \mathrm{~m}$ back run, and $20 \mathrm{~m}+10 \mathrm{~m}$ sharp stop turn. While doing basic football skills, we should also do well in the above skills training. The daily training is divided into two time courts in the morning and afternoon. The morning training is mainly based on strength training, supplemented by some basic football training. In the afternoon, football skills are the main focus of training, and physical ligaments should be moved. Before the start of the tournament, all the experimental subjects had the same training program and the same continuous training time every day. The athletes needed to try their best to complete all the training contents. Before the experiment, the 40 football players who participated in the experiment were tested for their physical fitness. The test results are recorded in Table 2.

According to data analysis, the agility of these football players is relatively low, mainly due to the unsmooth connection between the sudden turn and sudden stop and deceleration and reacceleration, and their lower limb explosive power and braking ability are also poor.

3.3. Experimental Process. All participants completed the measurement of maximum oxygen uptake capacity on the first day of the experiment. After 6 weeks, $3 \mathrm{ml}$ blood samples were collected from the cubital vein of the subjects on the day of vitamin $\mathrm{E}$ intake for testing. The control group only took starch capsules with similar appearance to vitamin E as placebo, which was often consistent with the normal experimental group. All subjects stopped the exercise training course after testing the exercise load and $\mathrm{VO}_{2}$ max and resumed the rest according to the rest day. In the quiet state, $3 \mathrm{ml}$ venous blood of athletes was extracted and placed for 2 hours at $3000 \mathrm{RPM}$, centrifuged for 5 minutes, and stored in a refrigerator at $-70^{\circ} \mathrm{C}$ until detected.

During the experiment, in order to eliminate the interference of food to the experimental results, for both the experimental group and the control group, the diet was carefully prepared and unified. Before the start of the exercise experiment, the experimenter will introduce the exercise mode and the matters needing attention during the exercise to all subjects in detail. It is especially emphasized that in the event of physical symptoms of maladjustment during the
TABLE 2: Sports fitness test results.

\begin{tabular}{lcc}
\hline Item & Mean & Standard deviation \\
\hline Jump in place $(\mathrm{m})$ & 0.36 & 0.27 \\
Standing long jump $(\mathrm{m})$ & 2.57 & 0.15 \\
Standing triple jump $(\mathrm{m})$ & 7.53 & 0.61 \\
10-meter sprint $(\mathrm{s})$ & 1.98 & 0.78 \\
20-meter sprint (s) & 3.3 & 0.11 \\
Four-way low hurdle jump (number) & 21.71 & 2.39 \\
YOYO test (m) & 777.17 & 92.87 \\
\hline
\end{tabular}

exercise experiment, the organizer should be immediately asked questions and be ready to terminate the exercise experiment at any time. All kinds of physiotherapy equipment are ready at any time in the laboratory to prevent all kinds of experimental accidents and ensure that the experiment is carried out in a safe environment.

After the end of the football league, the $\mathrm{C} 1$ and $\mathrm{T} 1$ groups were given aerobic exercise with maximum oxygen, while the $\mathrm{C} 2$ and $\mathrm{T} 2$ groups were given intermittent anaerobic exercise. In the $\mathrm{C} 1$ and $\mathrm{T} 1$ groups, $90 \% \mathrm{VO}_{2} \max$ intensity was used for exercise until exhaustion. The $\mathrm{C} 2$ and $\mathrm{T} 2$ groups ride full power bikes for 30 seconds $\times 3$ /interval for 3 minutes. Serum malondialdehyde (MDA), total antioxidant capacity (T-AOC), glutathione peroxidase (GSH-PX), and superoxide dismutase (SOD) were analyzed. MDA, SOD, and GSH-PX reagents were all provided by the Institute of Biological Engineering, and the tests were carried out strictly in accordance with the kit standard methods.

3.4. Mathematical Statistics. All experimental data were analyzed by SPSS 23.0, and the data were expressed in the form of mean \pm standard deviation. An independent sample $t$-test was used for comparison between the means of each group, and a paired $t$-test was used for comparison before and after themselves. The significance level was expressed as $P<0.05$ or $P<0.01$, with $P<0.05$ as significance level and $P<0.01$ as very significant.

\section{Experimental Results and Analysis}

4.1. Effects of Vitamin E Supplementation on Antioxidant Capacity of the Body. The effects of vitamin E supplementation on the antioxidant capacity of the body in the quiet state are shown in Table 3. It can be seen from the table that before vitamin E supplementation, there was no statistically significant difference in serum antioxidant levels between 
TABLE 3: Effects of vitamin E supplementation on antioxidant capacity of the body in quiet state.

\begin{tabular}{lccccc}
\hline Group & Status & SOD $(\mathrm{U} / \mathrm{ml})$ & GSX-PX $(\mathrm{U})$ & T-AOC $(\mathrm{U} / \mathrm{ml})$ & MDA $(\mathrm{nmol} / \mathrm{ml})$ \\
\hline \multirow{2}{*}{ Control group $(n=20)$} & Before taking & $76.84 \pm 22.24$ & $183.65 \pm 33.05$ & $10.92 \pm 3.46$ & $4.06 \pm 0.46$ \\
& After taking & $76.80 \pm 15.38$ & $185.09 \pm 35.58$ & $10.83 \pm 3.88$ & $4.88 \pm 0.95$ \\
\hline \multirow{2}{*}{ Experimental group $(n=20)$} & Before taking & $72.11 \pm 18.08$ & $185.71 \pm 34.92$ & $10.59 \pm 3.25$ & $4.80 \pm 0.34$ \\
& After taking & $87.75 \pm 17.72$ & $217.39 \pm 36.55$ & $13.14 \pm 4.17$ & $3.40 \pm 0.49$ \\
\hline
\end{tabular}

Changes of serum SOD before and after exercise

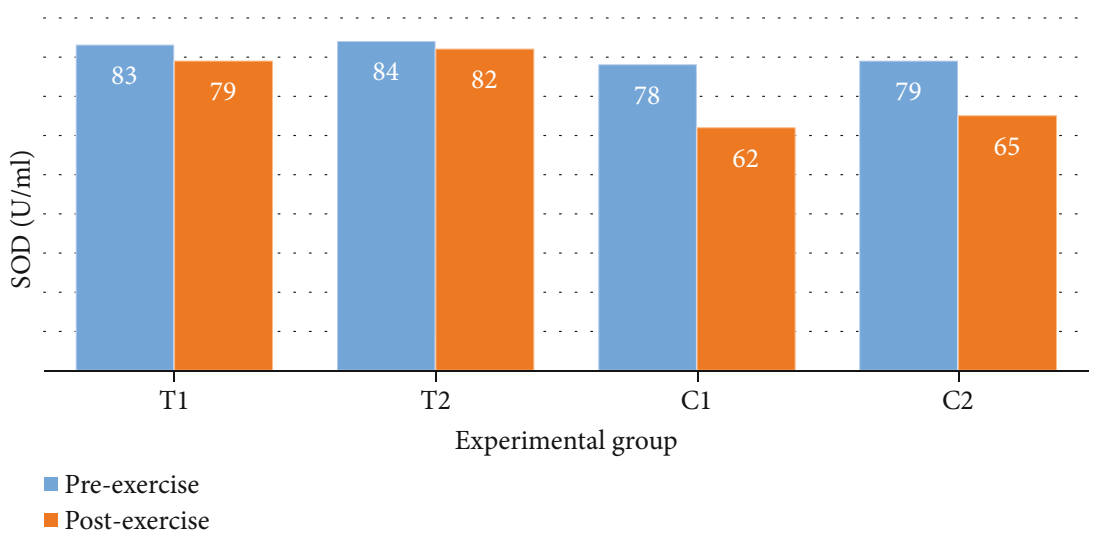

FIgure 3: Changes of serum SOD before and after exercise.

the experimental group and the control group, nor was there statistically significant difference in serum antioxidant levels between the control group before and after taking the same amount of placebo.

4.1.1. Changes of Serum SOD before and after Exercise. As can be seen from Table 3, after taking vitamin $\mathrm{E}$ for a period of time, the mean value of SOD in the serum of the experimental group increased from $72.11 \pm 18.08(\mathrm{U} / \mathrm{ml})$ to $87.75 \pm 17.72(\mathrm{U} / \mathrm{m} 1)$, with a significant increase $(P<0.05$ ). Compared with the experimental group, the mean value of serum SOD of subjects in the control group before and after taking the placebo was $76.84 \pm 22.24(\mathrm{U} / \mathrm{ml})$ and $76.80 \pm 15.38(\mathrm{U} / \mathrm{ml})$, respectively, with no significant difference between the two groups. It is worth noting that the mean value of serum SOD in the experimental group and the control group was $87.89 \pm 17.94(\mathrm{U} / \mathrm{ml})$, and the mean value of serum SOD in the control group was $76.23 \pm$ $15.86(\mathrm{U} / \mathrm{ml})$. Obviously, the mean value of serum SOD in the experimental group was significantly different from that in the control group $(P<0.05)$.

The changes of serum SOD before and after exercise are shown in Figure 3. It can be seen from the figure that the serum SOD value of all subjects decreased immediately after exercise, but there was no significant difference in the comparison data before and after exercise $(P>0.05)$. In general, the SOD value of the experimental group decreased relatively little while the exercise load was relatively large. In addition, the serum SOD value of the experimental group after exercise was significantly higher than that of the con- trol group before exercise and that of the control group after exercise.

4.1.2. Changes of Serum GSH-PX before and after Exercise. The data in Table 3 show that after taking vitamin $E$ for a period of time, there is a significant difference between the experimental group and the control group when taking placebo. The mean value of serum GSH-PX before and after taking vitamin $\mathrm{E}$ increased from $185.56 \pm 35.89$ (U) to $217.19 \pm 36.09(\mathrm{U})$, with a significant increase $(P<0.01)$. The mean values of serum GSH-PX in the control group after two weeks of placebo were $183.65 \pm 33.05$ (U) and $185.09 \pm 35.58(\mathrm{U})$, respectively, with no statistical difference between the two groups. The mean values of GSH-PX in the experimental group were $217.39 \pm 36.55(\mathrm{U})$ and $185.71 \pm$ $34.92(\mathrm{U})$, respectively, after two weeks of taking lycopene capsule compared with the control group after two weeks of taking placebo. The mean values of GSH-PX in the experimental group were significantly higher than those in the control group $(P<0.01)$.

The changes of serum GSH-PX before and after exercise are shown in Figure 4. As can be seen from the figure, compared with the C1 group, the GSH-PX value in the serum of the $\mathrm{T} 1$ group was significantly higher than that of the $\mathrm{C} 1$ group after the high-intensity aerobic exercise. Comparing the T2 group with the $\mathrm{C} 2$ group, it was found that after intermittent anaerobic exercise, the serum GSH-PX of the C2 group was significantly lower than that of the T2 group $(P<0.01)$. No matter in group T1 or group T2, the activity of GSH-PX in serum decreased immediately after exercise 
Changes of serum GSH-PX before and after exercise

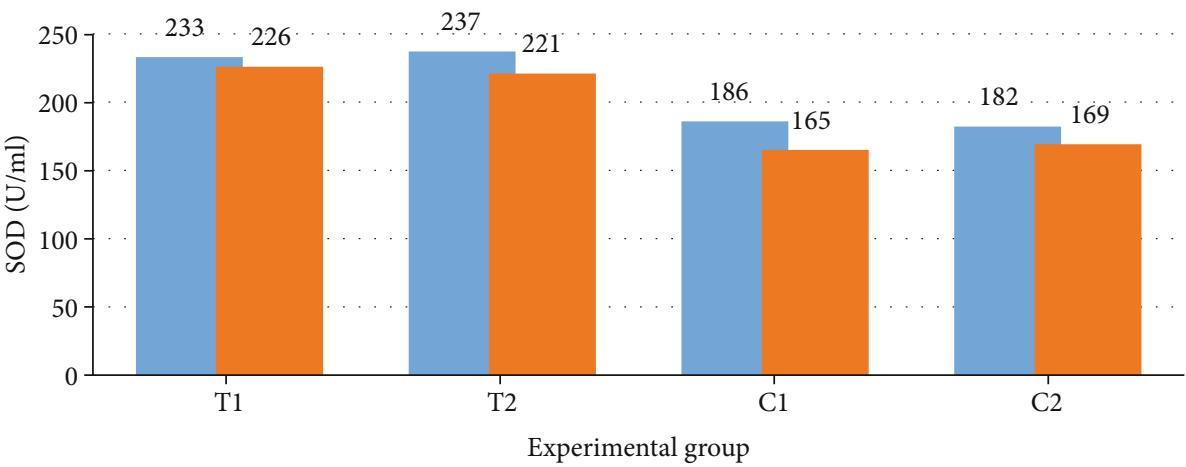

- Pre-exercise

- Post-exercise

FIGURE 4: Changes of serum GSH-PX before and after exercise.

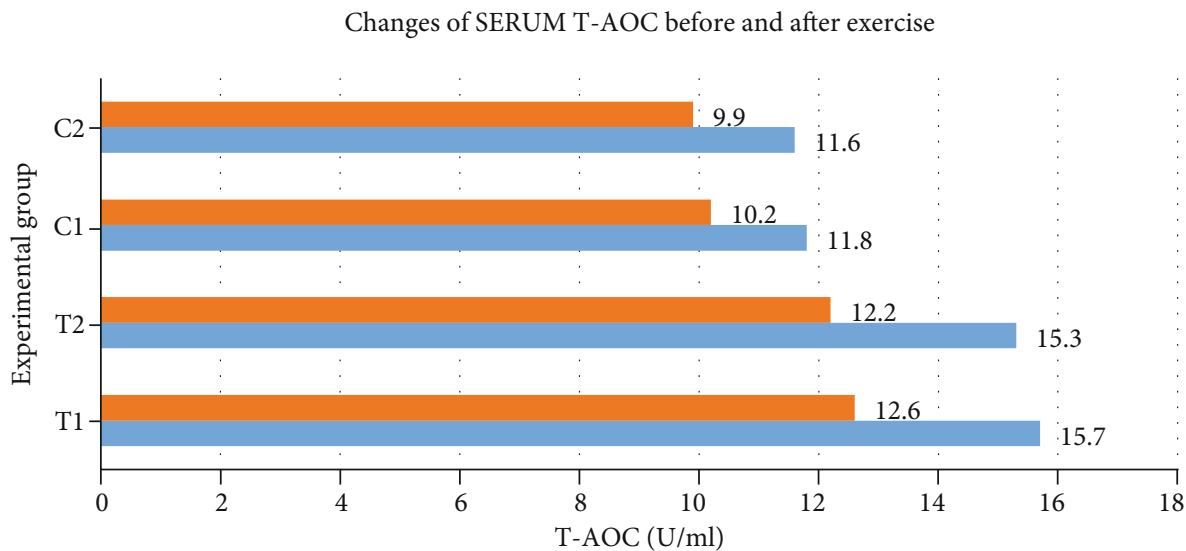

Post-Exercise

- Pre-Exercise

Figure 5: Changes of serum T-AOC before and after exercise.

load, but the activity of GSH-PX in the experimental group decreased slightly, and the difference was not statistically significant $(P>0.05)$.

4.1.3. Changes of Serum T-AOC before and after Exercise. The experimental results in Table 3 show that after taking vitamin $\mathrm{E}$ and placebo for a period of time, the mean values of serum T-AOC in the experimental group and the control group were $13.42 \pm 4.25(\mathrm{U} / \mathrm{ml})$ and $10.27 \pm 3.67(\mathrm{U} / \mathrm{ml})$, respectively. The serum T-AOC content in the experimental group was significantly higher than that in the control group $(P<0.05)$. In terms of total antioxidant capacity, the mean values of serum T-AOC of the control group before and after taking placebo for a period of time were $10.92 \pm 3.46$ and $10.83 \pm 3.88(\mathrm{U} / \mathrm{ml})$, respectively, with no statistical difference between the two groups. The mean value of serum T-AOC of the experimental group before and after taking vitamin $\mathrm{E}$ for a period of time increased from $10.59 \pm 3.25$ $(\mathrm{U} / \mathrm{ml})$ before taking to $13.14 \pm 4.17(\mathrm{U} / \mathrm{ml})$, with a significant increase $(P<0.05)$.

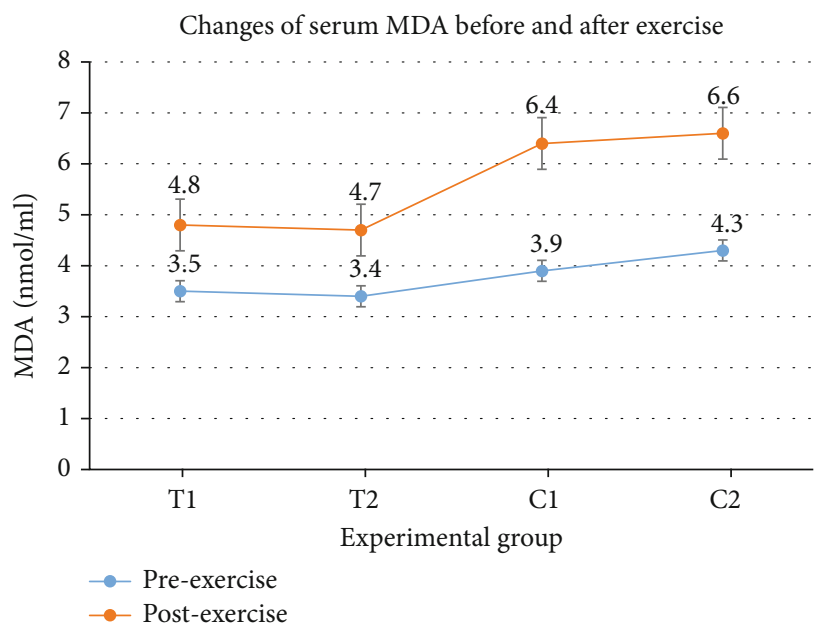

FIGURE 6: Changes of serum MDA before and after exercise. 


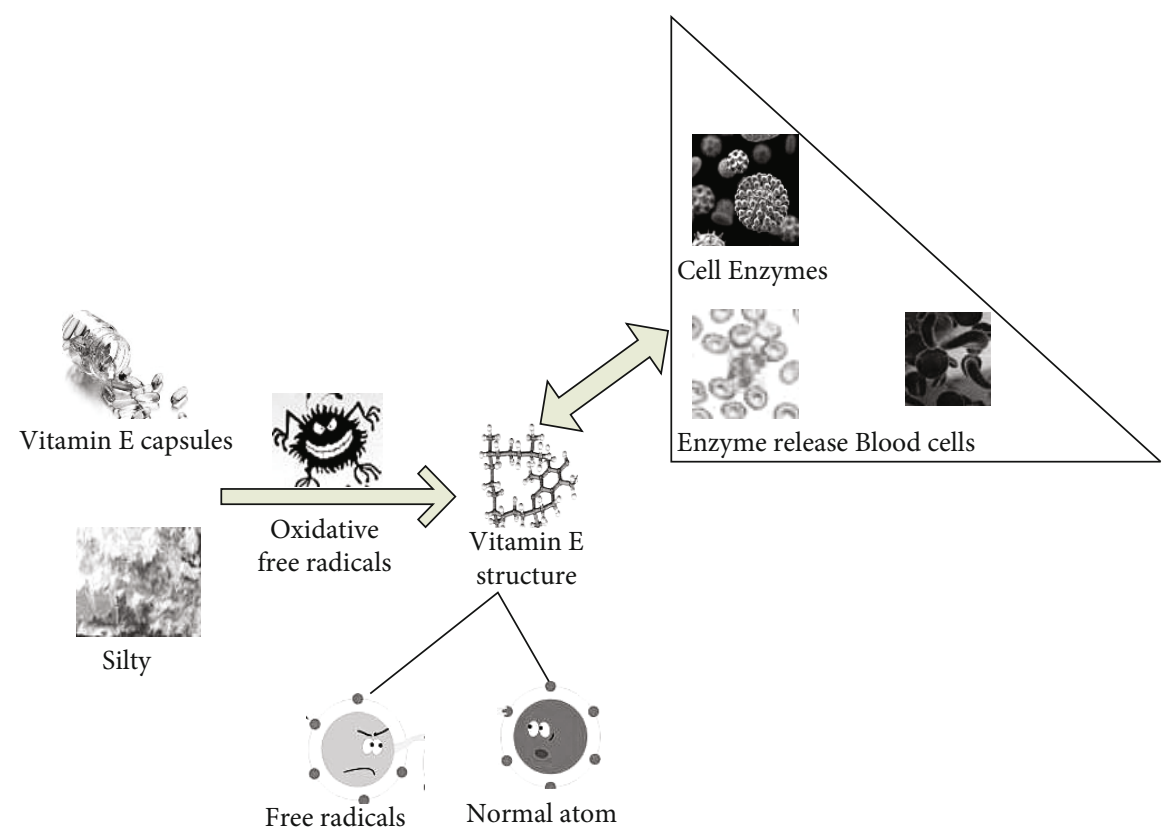

FIgURE 7: Vitamin E and free radical oxidation.

The changes of serum T-AOC before and after exercise are shown in Figure 5. It can be found from the figure that there is almost no difference in serum T-AOC value between the two exercise modes in the experimental group, and the serum T-AOC value after exercise decreases compared with that before exercise. The total antioxidant capacity of serum of all subjects immediately after exercise was significantly decreased compared with that before exercise. In addition, the serum T-AOC values of the experimental group after exercise were higher than those of the control group before exercise.

4.1.4. Changes of Serum MDA before and after Exercise. For the control of all the subjects in the placebo for some time, in the serum MDA, there was no significant difference compared with taking before4.06 \pm 0.46 and $4.88 \pm 0.95(\mathrm{~nm} / \mathrm{ml})$; for the experimental group under the action of vitamin $\mathrm{E}$, serum MDA before taking with an average of $4.80 \pm 0.34$ $(\mathrm{nmol} / \mathrm{ml}$ ) was reduced to $3.40 \pm 0.49 \mathrm{nmol} / \mathrm{ml}$, with an obvious reduction and significant difference $(P<0.05)$. In addition, the comparison results between the control group taking placebo and the experimental group taking vitamin $\mathrm{E}$ for a period of time showed that the mean value of MDA was $3.25 \pm 0.31(\mathrm{~nm} / \mathrm{ml})$ and $4.20 \pm 0.78(\mathrm{~nm} / \mathrm{ml})$, respectively. The mean value of $\mathrm{MDA}$ in the experimental group was significantly lower than that in the control group, with significant statistical difference $(P<0.05)$.

The changes of serum MDA before and after exercise are shown in Figure 6. As can be seen from the figure, MDA values in the $\mathrm{C} 1$ group of the control group were significantly increased after exercise, and the difference was significant $(P<0.01)$; MDA values in the $\mathrm{C} 2$ group of the control group were also significantly increased after exercise $(P<0.01)$. The serum MDA values in the experimental group after T1 and T2 exercise were also increased compared with those before exercise, but the increase was small, and there was no statistical difference $(P>0.05)$. There was no statistically significant difference between the two types of exercise.

4.2. Effects of Vitamin E Supplementation on Free Radicals. $\mathrm{MDA}$ is a representative product of lipid peroxidation and also a sensitive index to measure whether the free radical metabolism of the body exceeds the limit. The content index of MDA in blood can objectively reflect the level of free radicals produced by the body and indirectly reflect the ability of the body to eliminate more free radicals. When there are too many free radicals in the body, the ability of lycopene with a higher concentration to scavenge free radicals and the antioxidant system of the body are relatively insufficient, which will lead to the attack of free radicals on cells. The excess free radicals will promote the production of lipid peroxidation, especially the change of cell genetic material, further accelerating the aging of body cells and reducing the activity of cell enzymes. The reaction performance of vitamin $\mathrm{E}$ and free radicals in athletes is shown in Figure 7.

The change of cell enzyme activity can directly and indirectly reflect the free radical reaction in organisms. In this experiment, the activity of SOD and GSH-PX and the ability of T-AOC were decreased, which may be due to the large increase of free radical oxidase in cell tissues for selfprotection of the brain, kidney, and other important tissues after exercise. Therefore, a large amount of antioxidant enzymes will be consumed in skeletal muscles and other important organs. This also indicates that the lipid peroxidation in the body increases, and mitochondria and cell membrane suffer more serious damage. The model for football 


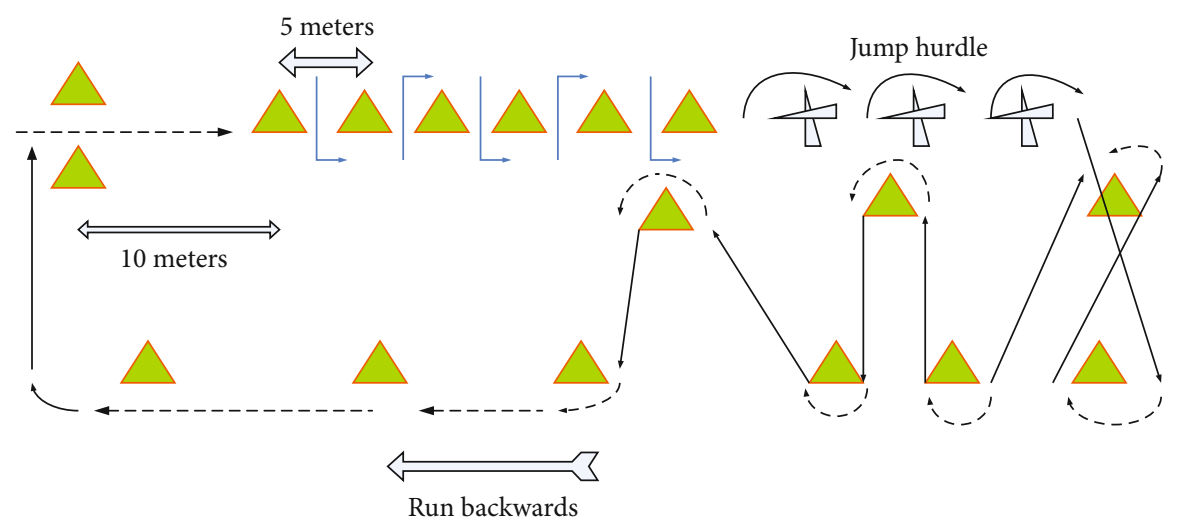

FIGURE 8: Special endurance training.

TABle 4: Physical recovery data.

\begin{tabular}{lccc}
\hline The main nature of the load & Original energy supply capacity & Solution energy supply capacity & Aerobic energy supply capacity \\
\hline Original energy load & 36 & 18 & $4-8$ \\
Solution energy load & 18 & $36-54$ & $4-8$ \\
Aerobic energy load & 4 & $18-36$ & 54 \\
\hline
\end{tabular}

TABLE 5: Athletes' heart rate and lactose records.

\begin{tabular}{|c|c|c|c|c|}
\hline & $11 \mathrm{~km} / \mathrm{h}$ & $12 \mathrm{~km} / \mathrm{h}$ & $13 \mathrm{~km} / \mathrm{h}$ & $14 \mathrm{~km} / \mathrm{h}$ \\
\hline Control lactic acid & $2.23 \pm 0.39$ & $3.12 \pm 0.78$ & $4.97 \pm 1.21$ & $5.71 \pm 1.27$ \\
\hline Lactic acid in experimental group & $2.12 \pm 0.19$ & $3.21 \pm 0.57$ & $5.01 \pm 0.72$ & $5.89 \pm 0.77$ \\
\hline Control heart rate & $157 \pm 6$ & $161 \pm 8$ & $176 \pm 7$ & $181 \pm 11$ \\
\hline Heart rate of experimental group & $158 \pm 6$ & $171 \pm 8$ & $177 \pm 9$ & $183 \pm 8$ \\
\hline
\end{tabular}

players' special endurance dribbling training is constructed, as shown in Figure 8.

The interval between each item is 20 seconds, the number of exercises is 4 cycles, and the interval between groups is 6 minutes.

The time required for physical recovery of athletes with different types of load training is recorded, as shown in Table 4.

The experimental results of this paper suggest that vitamin $\mathrm{E}$ supplementation can reduce MDA production, and the consistency of the control group further confirms the significant reduction of MDA. Previous animal experiments have shown that MDA levels in rats are affected by the secretion of monoamine oxidase, while vitamin $\mathrm{E}$ can reduce MDA levels in rats. We suspect that it is caused by the anaerobic exercise training unit of the body's oxygen consumption increasing, leading to the formation of oxygen free radicals, and antioxidant vitamin $\mathrm{E}$ can consume antioxidant enzyme activity, if in the process of vitamin $\mathrm{E}$, the activity of antioxidant enzymes within tissues rises, so vitamin E supplements can reduce the generation of MDA and prevent and reduce the dynamic free radical damage.
4.3. Effects of Vitamin E and Acute Anaerobic Exercise on Human Free Radical Metabolism. In previous studies, anaerobic exercise as a means of exercise is not much studied; most of the exhaustion exercise as a means of exercise is used to observe the metabolism of free radicals. Therefore, there are few reports on the effects of anaerobic exercise on free radical metabolism and antioxidant enzymes. The ball activity under high-intensity physical confrontation, various speed changes, direction changes, and man-to-man defense are the main factors that cause the high load intensity of the game. For this reason, the athletes have launched a record of these factors. The data is shown in Table 5 .

In this study, two different acute modes of exercise were simulated by means of $90 \% \mathrm{VO}_{2} \max$ intensity exercise to exhaustion and full power cycling for 30 seconds $\times 3$ interval for 3 minutes. Both methods were found to increase the production of free radicals in the body. Two types of movement after the test, where the average heart rate of subjects is more than 120 times per minute, suggest that even large intensity of acute exercise which has shorter duration, as long as it reached a certain strength, can also cause increased production of free radicals in 


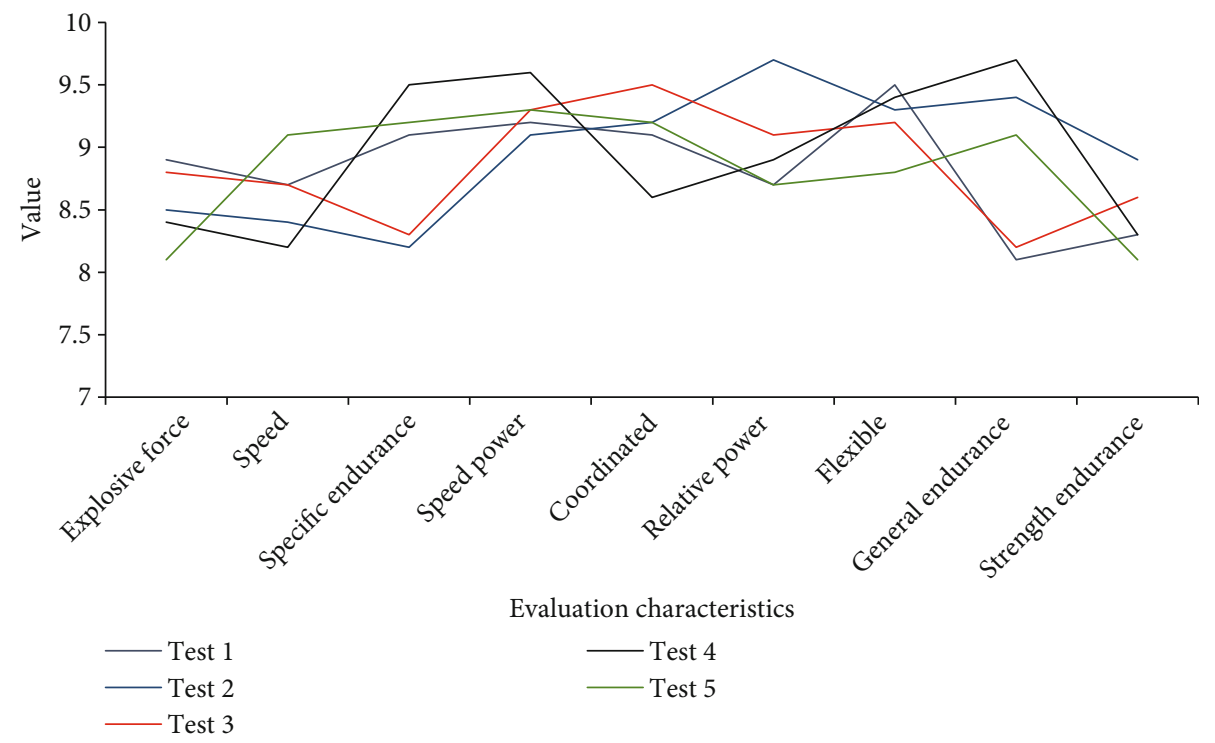

Figure 9: Physical fitness test of athletes.

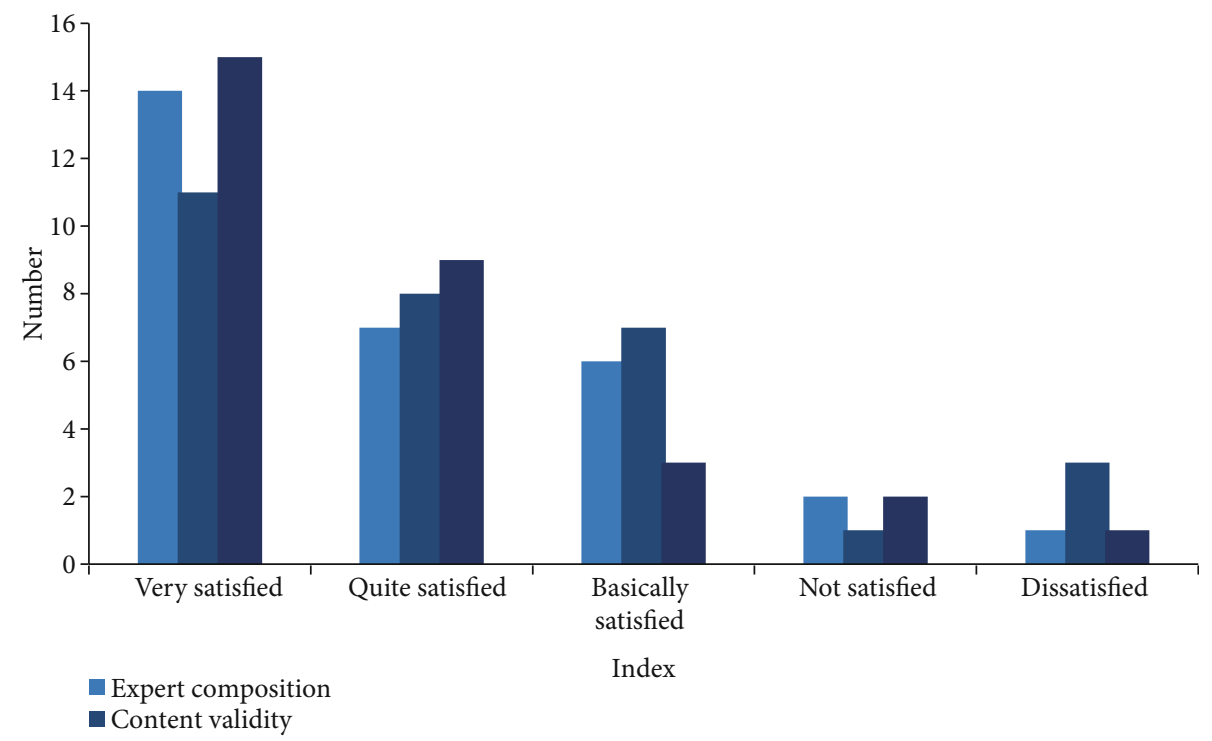

FIgURE 10: Questionnaire survey results of experts and coaches.

the body; for movement after the steady phase as well, it is difficult to restore to the normal expression level in a short time.

After supplementing the athletes with a certain amount of vitamins, another 5 rounds of tests were carried out, and the experts evaluated the various physical reaction times of these athletes (1-10). The experimental results are shown in Figure 9.

In terms of vitamin $\mathrm{E}$ supplementation, the $\mathrm{T} 1$ group and the $\mathrm{C} 1$ group were given exercise in the same way in this study. Although the T1 group was supplemented with vitamin E, the exercise time of the T1 group was only slightly longer than that of the $\mathrm{C} 1$ group, and the difference was not statistically significant. On the whole, there was no sig- nificant difference between the experimental group and the control group in terms of exercise time. Therefore, there is still no direct and effective evidence to prove whether vitamin E supplement can replenish physical strength for the body.

In order to evaluate the physical fitness of these athletes, 30 experts and coaches were invited to conduct a questionnaire survey. The results of the survey are shown in Figure 10.

The data in Figure 10 shows that $90 \%$ of the experts are satisfied with the content arrangement of the test, and $90 \%$ of the experts believe that the structure of the test is satisfied, so the validity of this study can be considered to be relatively high. 


\section{Conclusion}

In this study, the experimental group was given vitamin E, while the control group was given a placebo without any vitamin, and the experimental group and the control group were divided into aerobic exercise and anaerobic intermittent exercise to study the effect of vitamin E supplementation on free radicals and their physical strength of football players. By analyzing the changes of malonaldehyde (MDA), total antioxidant capacity (T-AOC), superoxide dismutase (SOD), and glutathione peroxidase (GSH-PX) in soccer players before and after soccer training, this study is aimed at exploring the degree of vitamin E supplementation on free radical metabolism and antioxidant enzyme activity of soccer players. The main conclusions of the study are as follows:

(1) Vitamin E can significantly reduce the production of free radicals under the intermittent anaerobic exercise of football players and improve the antioxidant capacity of the body

(2) Even if the duration of high-intensity acute exercise is relatively short, as long as it reaches a certain intensity, it will also cause an increase in the production of free radicals in the body

(3) Whether vitamin E supplement can replenish physical strength for the body still lacks direct and effective evidence to prove

\section{Data Availability}

Data sharing is not applicable to this article as no datasets were generated or analyzed during the current study.

\section{Conflicts of Interest}

The author declares that they have no conflicts of interest.

\section{References}

[1] A. R. Goswami and T. Ghosh, "Vitamin E reduces hypobaric hypoxia-induced immune responses in male rats," High Altitude Medicine \& Biology, vol. 20, no. 1, pp. 12-21, 2019.

[2] E. Chikvaidze and M. Topeshashvili, "Effect of ascorbic acid (vitamin C) on the ESR spectra of the red and black hair: pheomelanin free radicals are not always present in red hair," Magnetic Resonance in Chemistry, vol. 53, no. 12, pp. 1019-1023, 2015.

[3] Z. Kiasalari, M. Khalili, S. Shafiee, and M. Roghani, "The effect of vitamin $\mathrm{E}$ on learning and memory deficits in intrahippocampal kainate-induced temporal lobe epilepsy in rats," Indian Journal of Pharmacology, vol. 48, no. 1, pp. 11-14, 2016.

[4] S. L. Rowell and O. K. Muratoglu, "Investigation of surgically retrieved, vitamin E-stabilized, crosslinked UHMWPE implants after short-termin vivoservice," Journal of Biomedical Materials Research Part B Applied Biomaterials, vol. 104, no. 6, pp. 1132-1140, 2016.

[5] A. Rostami, S. A. Moosavi, and V. Changizi, "Radioprotective effects of selenium and vitamin-E against $6 \mathrm{MV} \mathrm{X}$-rays in human blood lymphocytes by micronucleus assay," Medical Journal of the Islamic Republic of Iran, vol. 30, no. 1, p. 367, 2016.

[6] K. Cordero, G. G. Coronel, M. Serrano-Illán, J. Cruz-Bracero, J. Figueroa, and Marino De León, "Effects of dietary vitamin E supplementation in bladder function and spasticity during spinal cord injury," Brain Sciences, vol. 8, no. 3, p. 38, 2018.

[7] Y. Okamura, A. Omori, N. Asada, and A. Ono, "Effects of vitamin $\mathrm{C}$ and $\mathrm{E}$ on toxic action of alcohol on partial hepatectomy-induced liver regeneration in rats," Journal of Clinical Biochemistry and Nutrition, vol. 63, no. 1, pp. 50-57, 2018.

[8] R. Zulkapli, F. Abdul Razak, and R. B. Zain, "Vitamin E ( $\alpha$ tocopherol) exhibits antitumour activity on oral squamous carcinoma cells ORL-48," Integrative Cancer Therapies, vol. 16, no. 3, pp. 414-425, 2017.

[9] M. M. Fagan, R. Pazdro, J. A. Call et al., "Assessment of oxidative stress and muscle damage in exercising horses in response to level and form of vitamin E," Journal of Equine Veterinary Science, vol. 52, no. 1, pp. 80-81, 2017.

[10] A. A. Ghanbari, K. Shabani, and D. Mohammad Nejad, "Protective effects of vitamin E consumption against 3MT electromagnetic field effects on oxidative parameters in substantia nigra in rats," Basic \& Clinical Neuroence, vol. 7, no. 4, pp. 315-322, 2016.

[11] K. Aleksandra, K. Beata, and P. Kamila, "Relationship between the degree of antioxidant protection and the level of malondialdehyde in high-performance Polish Holstein-Friesian cows in peak of lactation," Plos One, vol. 13, no. 3, article e0193512, 2018.

[12] J. Cedrowski, G. Litwinienko, A. Baschieri, and R. Amorati, "Hydroperoxyl radicals (HOO.): vitamin E regeneration and H-bond effects on the hydrogen atom transfer," Chemistry, vol. 22, no. 46, pp. 16441-16445, 2016.

[13] Y. Li, J. Zhao, Z. Lv, and J. Li, "Medical image fusion method by deep learning," International Journal of Cognitive Computing in Engineering, vol. 2, pp. 21-29, 2021. 